\title{
Hepatitis C Antiviral Therapy In Patients With Successfully Treated Hepatocellular Carcinoma: Dancing With Wolves
}

This article was published in the following Dove Press journal: Journal of Hepatocellular Carcinoma

\author{
Mohamed El Kassas (iD) \\ Ahmed Tawheed (iD) \\ Mohamed Eltabbakh (iD) ${ }^{2}$ \\ Ahmed Kaseb ${ }^{3}$ \\ 'Endemic Medicine Department, Faculty \\ of Medicine, Helwan University, Cairo, \\ Egypt; ${ }^{2}$ Tropical Medicine Department, \\ Faculty of Medicine, Ain Shams \\ University, Cairo, Egypt; ${ }^{3}$ Department of \\ Gastrointestinal Medical Oncology, The \\ University of Texas M.D. Anderson \\ Cancer Center, Houston, TX, USA
}

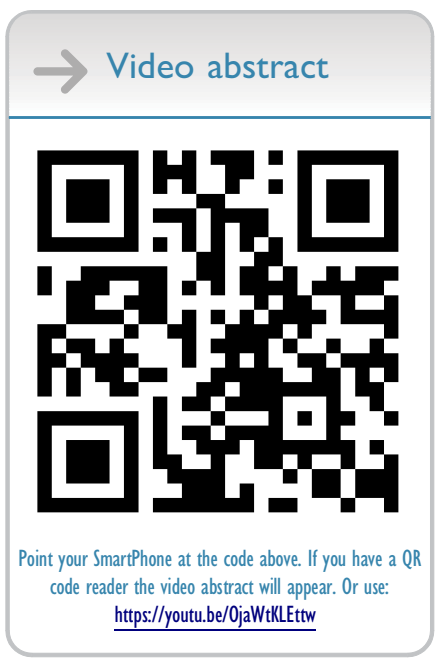

Correspondence: Mohamed El Kassas Endemic Medicine Department, Faculty of Medicine, Helwan University, Cairo, Egypt Tel +20 I I I 4455552

Fax +20227239227

Email m_elkassas@yahoo.com

\begin{abstract}
Hepatitis C virus (HCV) infection is known to be one of the leading causes of hepatocellular carcinoma (HCC) all over the world. Previously, multiple studies have confirmed a decreased rate of HCC occurrence or recurrence in the cases of hepatitis $\mathrm{C}$ associated cirrhosis after treatment with interferon, in comparison to the untreated cases, even in the absence of clearance of HCV. Treatment programs with direct-acting antivirals (DAAs) as a new method for HCV treatment and cure in 2014, with higher safety and efficacy, were considered as an important step in the treatment of patients with history of HCC, improving their overall prognosis. Recently, reports coming from various European centers claimed that the risk of HCC increased following DAAs therapy, especially in cases with previous HCC. Moreover, other studies revealed that the recurrence of HCC after DAAs treatment was more aggressive. Even though others were not able to conclude the same results, the role of DAA therapy in recurrence of HCC in patients with previous HCC after sustained virological response (SVR) achievement remains questionable. This review explored the existing literature and discussed opinions on the possibility of increasing recurrence of HCC following DAA therapy, possible mechanisms, predictors of HCC recurrence post DAAs, and whether those patients should be treated or not.
\end{abstract}

Keywords: hepatitis $\mathrm{C}$ virus, hepatocellular carcinoma, recurrence, direct-acting antiviral agents

\section{Introduction}

Hepatocellular carcinoma (HCC) is considered the sixth most common cancer and the third most common reason of death among different types of cancers all over the world. Liver cancer among Egyptian patients constitutes $1.68 \%$ of all the cancers, whereas HCC constitutes $70.48 \%$ of all hepatic cancers. ${ }^{1}$ During the era where interferon (IFN) was used for Hepatitis C virus (HCV) treatment, many studies found that sustained virological response (SVR) after IFN treatment is a direct cause of decreased incidence of HCC and increased survival rates. ${ }^{2}$

The beginning of treatment programs in 2014, with new direct-acting antivirals (DAAs) having higher safety and efficacy, was considered an important and new step in the management of HCC treated patients, in order to improve their prognosis. However, various European research centers recently claimed that the risk of HCC recurrence was increased following DAAs therapy, especially in cases with previous HCC. ${ }^{3}$ Others have revealed that the pattern of HCC recurrence following DAAs therapy was more aggressive. ${ }^{4,5}$ 
The one-year risk of HCC recurrence following curative intervention varies from $25-30 \%$ in various studies, and this increases up to $75 \%$ after 5 years of intervention in some studies. ${ }^{6,7}$

Taking this data into account, the usage of DAAs in cases with previous $\mathrm{HCC}$ can be doubtful. Therefore, in this review, we will discuss whether HCC patients should be treated with DAAs or not and the predictors of HCC recurrence post DAAs.

\section{HCC Recurrence Risk After Interferon And Ribavirin Therapy}

Several studies confirmed that treatment of HCV patients with combination of pegylated interferon and ribavirin (PEG-IFN/RBV) can generally improve their survival and prognosis by decreasing the risk of HCC development during follow-up. ${ }^{8}$ This finding paved the way for its use to reduce the risk of recurrence of HCC following its curative intervention. Several specific studies and metaanalyses suggested that previous exposure to IFN/RBV can reduce the risk of HCC recurrence following curative intervention. In 2018, an interesting report from China declared that ribavirin may have inhibitory effect on the growth of HCC by affecting some miRNAs, especially miR-96, which is responsible for increasing cell proliferation and invasion in HCC. ${ }^{9}$ Another explanation is the direct immunostimulatory and antitumor effects of IFN, which could reduce the risk of development of HCC in cases with SVR following IFN treatment regimens when comparing them with non-responders. ${ }^{2,10-12}$

The kickoff came from a multicenter Japanese study including 2890 chronic $\mathrm{HCV}$ patients. Of these, 2400 patients were treated with IFN, while the remaining patients were left untreated. HCC development was noted in 89 cases receiving IFN versus 59 cases not receiving treatment. The annual HCC incidence showed a proportional increase in the degree of liver fibrosis reaching $7.9 \%$ between cases with $\mathrm{F} 4$ fibrosis stage in comparison to $0.5 \%$ in cases with $\mathrm{F} 0$ and F1 fibrosis stage. There was a significant difference in cumulative incidence in both treated and not-treated cases: cases with F2 stage of fibrosis $(p=0.0128)$ and cases with F3 $(\mathrm{p}=0.0011) .{ }^{13}$

In 2001, another pilot study by Takeaki u et al showed that local HCC recurrence was $6 \%$ in cases treated with IFN and $9 \%$ in cases not receiving IFN, which was, however, insignificant. The distant recurrence of HCC decreased in the group receiving IFN (22\%) as compared to the group that did not receive IFN (82\%) and was, thus, highly significant. ${ }^{14}$ Also, Arnaoutakis et al revealed that the incidence of HCC decreased significantly in cases with SVR after IFN therapy compared to non-IFN group (48 vs $70 \%$, respectively). ${ }^{7}$ Similar findings were confirmed by Naoya et al and Cheol et al who reported that the recurrence of HCC decreased significantly among SVR cases as compared to those not achieving SVR and control group. ${ }^{15,16}$ A metaanalysis by Miyake et al concluded that treating $\mathrm{HCV}$ with IFN $\alpha$ and RBV after curative intervention for $\mathrm{HCC}$ inside the criteria of Milan could be of value in the prevention of HCC recurrence. ${ }^{12}$

A huge study from the United States that included a large number of cases $(33,005$ patients) treated with IFN therapy confirmed the protective role of IFN against HCC. The measured outcome was the incidence of HCC in cases achieving SVR in comparison to cases not achieving SVR. The annual HCC incidence rate in cases with SVR was $3.27 / 1000$ patients-years in contrast to $1.32 / 100$ patientsyears in cases without SVR. There was higher HCC risk among patients with cirrhotic state, older age, diabetes, and genotype $3 .{ }^{17}$

On the contrary, some studies reported that $\mathrm{HCC}$ recurrence rate was not significantly different between IFN group and non-IFN group. ${ }^{2,18}$

Concluding, from the cumulative evidence for $\mathrm{HCV}$ during the IFN therapy era, there was a relatively solid background to support the role of viral eradication in preventing HCC recurrence. Age, fibrosis, and SVR were highly associated with the risk of HCC recurrence; this was always a constant finding despite the use of IFN regimen, the type of HCC curative treatment or the geographical region.

\section{HCC In The Era Of Treatment With DAAs}

The introduction of new DAAs led to a revolution in HCV management and marvelous increase in SVR levels. ${ }^{19}$ Despite the high safety margin of DAAs therapy which enabled treatment of advanced cases, ${ }^{20}$ and with expected lower recurrence rate of HCC post-treatment, there were some contradictory reports on HCC recurrence rates post SVR.

\section{DAAs Increase HCC Recurrence Rate?}

Researchers were interested in exploring the possibility of increase in occurrence and recurrence of HCC, after SVR was achieved with DAAs. A Spanish cohort study from 
Reig et al in 2016 included 58 HCC cases treated either with curative or palliative measures who then received DAAs. The study showed a high rate of recurrence (27.6\%) after 5.7 months of median follow-up. Minimizing the time between HCC intervention and beginning of antiviral treatment was considered to be associated with higher rate of HCC recurrence $(41.2 \%){ }^{3}$ Following an increasing number of patients with longer follow-up period, the results validated the initial observation and suggested a more aggressive course of HCC recurrence with a rapid rate of tumor progression. ${ }^{4,21}$

A similar trend was described by Conti et al, where 59 cases with history of $\mathrm{HCC}$ were included, with recurrence of HCC in 17 cases after DAAs therapy (28.8\%) and with a median period of follow-up of 5.5 months. Age of the patients and the degree of fibrosis were considered to be associated with a high rate of recurrence. ${ }^{22}$

Further, two consecutive publications of retrospective nature reported that a higher recurrence rate following DAAs therapy was noticed among the cases who underwent treatment for HCC or liver transplants for the same cause. $^{23,24}$ Kolly et al demonstrated that disease-free survival rate of HCC in six months following DAAs treatment was $77 \%{ }^{23}$ Recurrence was associated with the period between HCC intervention and the beginning of antiviral treatment. ${ }^{23}$ Yang et al demonstrated the higher risk of HCC recurrence in cases awaiting liver transplantation and receiving DAA treatment. ${ }^{24}$ The recurrence rate was higher in recipients who received DAA treatment $(27.8 \%$ vs $9.5 \%)$ and occurred shortly following liver transplant ( $<6$ months) in most recipients. ${ }^{24}$

A similar conclusion was demonstrated by El Kassas et al in a comparative study from Egypt. The recruited HCC cases with previous history of successful intervention were divided into two groups. The first group (53 cases) received DAAs treatment with a recurrence rate of $37.7 \%$, while the second group (63 cases), which did not receive DAAs, had a recurrence rate of $25.4 \%$. They concluded that DAAs can increase the risk of HCC recurrence by up to 4 times. ${ }^{5}$ In addition to being a comparative study, the point of strength in this study was that they included only cases with a successful HCC curative intervention method. ${ }^{5}$

Granata et al also reported that DAAs therapy did not decrease the risk of HCC recurrence, which was 11 out of 38 patients with SVR and recurrence was notable during treatment with DAAs in two patients. ${ }^{25}$

\section{DAAs Decrease HCC Recurrence Rate}

In contrast to the findings of the previous reports, Victor et al reported that among 68 cases of $\mathrm{HCV}$ with history of HCC, 23 patients received DAAs therapy while 45 did not. $^{26}$ The disease-free interval between HCC intervention and recurrence of $\mathrm{HCC}$ increased in cases receiving DAAs therapy, the median time between both the events was 17.4 months among patients treated with DAAs and 10.1 months among untreated patients. $^{26}$

Another supporting study in this direction was from Japan, by Hiroko et al including 83 cases, that received curable intervention for $\mathrm{HCC}$ either with resection or ablation. About 28 months was the median follow-up period from HCC intervention after receiving DAAs. The results of this study should be considered as they compared the HCC recurrence rate according to both virological response and regimen of treatment. They highlighted two important findings; firstly, the recurrence rate was more in patients who received IFN-based regimens and did not achieve SVR than in patients who achieved SVR (5 year incidence $77 \%$ to $47 \%$ ). In patients who received IFN-free regimens, the 3 year incidence was $22.9 \%$ in patients who achieved SVR versus $40 \%$ in those who did not. Secondly, the recurrence rates between IFN-based group in comparison to IFN-free group were not significantly different ( 5 year survival $54.2 \%$ to $45.1 \%$ ). ${ }^{27}$

Another Japanese study analyzed 270 patients with 20.7 months of median follow-up. A total of 61 cases of $\mathrm{HCC}$ recurrence were reported. They concluded that the treatment with DAA after successful HCC intervention reduced the recurrence rate significantly in comparison with untreated patients. ${ }^{28}$ However, a significantly high rate of recurrence of HCC was noted in HCC cases with history of previous intervention. ${ }^{28}$

Similarly, Sangiovanni et al concluded that SVR post IFN-free regimens can decrease the risk of HCC recurrence. In their study, the yearly recurrence rate was $29.7 \%$ in 120 cases, receiving DAAs following complete response to HCC intervention. ${ }^{29}$

\section{DAAs Do Not Affect HCC Recurrence Rate?}

In 2017, a prospective study by Cabibbo et al demonstrated that after treatment with DAAs, the HCC recurrence probability after six months and one year of treatment was $12 \%$ and $26.6 \%$, respectively, which is in accordance with the other studies. ${ }^{30}$ Early recurrence of 
HCC was associated independently with the size of tumor and the presence of previous recurrence. ${ }^{30}$ They reported that the early HCC recurrence risk following DAA therapy in previously treated $\mathrm{HCC}$ cases remained high but less than the risk of $\mathrm{HCC}$ recurrence in cases that did not receive DAAs treatment. ${ }^{30}$

Moreover, in a study done by Ogawa et al, DAAs therapy did not augment the risk of HCC recurrence. In this study, a total of 152 cases with previous HCC treatment who received DAA therapy with SVR after treatment, were followed. HCC recurrence occurred in $16.5 \%$ of patients (median follow-up 17 months). ${ }^{31}$ The authors also reported non-significant differences when they compared the cumulative recurrence of HCC in one year (23.1\%) in this cohort, with that of another cohort by the same group published in 2012, who received IFN and RBV (recurrence rate of $22.2 \%$ ). ${ }^{32}$ These results were limited by median age difference in both the groups; those who received IFN had median age of 57 years while those who received IFN-free DAAs had median age of 66 years. This difference could be explained by the treatment of more elderly patients with DAAs who were not eligible for IFN treatment in the past. ${ }^{31,32}$ These results were in concordance with Mashiba et al's study in which 368 patients received IFN-free regimens following curative intervention for HCC and 148 patients received IFN-based regimens. The recurrence rate was not significantly different between both the groups. ${ }^{33}$

\section{Meta-analyses And Systematic Reviews}

Regarding the analytical studies, the first was the ANRS study in 2016, which analyzed three cohorts. ${ }^{34}$ The first cohort was ANRS CO22 HEPATHER which had 267 cases with history of HCC. Among these, 189 cases received DAAs therapy while 78 cases did not, and the recurrence rates were $12.6 \%$ and $20.5 \%$ respectively. The second one was ANRS CO12 CirVir cohort which included 79 cases with previous HCC history. The third cohort was ANRS CO23 CUPILT cohort that included 314 cases who underwent liver transplant and had pretransplant HCC history, and all were treated with DAAs. Only 7 patients had HCC recurrence with a percentage of $2.22 \%$. However, the conclusion of this analytical study did not support the increased possibility of HCC recurrence hypothesis. This study had many strong points as it was a prospective study, with large number of enrolled patients, and included those cases who received only curative interventions for hepatocellular carcinoma. ${ }^{34}$
Waziry et al included forty-one studies with a total number of 13,875 patients in a meta-analysis and addressed the issue of HCC recurrence and occurrence, during IFN and DAAs eras. There were 17 studies which discussed HCC recurrence (DAA: 10, IFN: 7; retrospective: 5, prospective: 11, and retrospective-prospective: 1$).{ }^{35}$ Also, there was shorter median follow-up period in the previous studies (about 1.3 vs 5 years) and there was nearly the same age in IFN studies compared to DAA studies (66 vs 64 years). In IFN studies, the recurrence of HCC was about 9.21/100 per year in comparison to $12.16 / 100$ per year in DAA studies. DAA treatment was not associated with higher HCC recurrence after adjusting the age and study period of follow-up (95\% CI 0.11-3.45, p: 0.56). ${ }^{35}$

A meta-analysis by Saraiya et al included and analyzed the results of 26 studies. ${ }^{36}$ Out of these 24 studies including 1820 patients, DAAs treatment was associated with recurrence of $\mathrm{HCC}$ ranging from $0 \%$ to $59 \%$ (with estimated pool of $24.4 \%, 95 \%$ CI: $18.4 \%-30.4 \%$ ). Recurrence of HCC was $21.9 \%$ in 11 manuscripts with $95 \%$ CI: $16.2 \%-28.3 \%$. There was an association between HCC recurrence after DAAs treatment and previous $\mathrm{HCC}$ recurrence history before treatment and a short period between HCC response and DAA initiation. An acceptable rate of $\mathrm{HCC}$ recurrence following DAA treatment was reported, especially if DAA treatment was postponed by six months following complete response of HCC. Limitations of this study included heterogeneity of cohorts, shorter durations of follow-up, and absence of HCC classification before starting DAAs which could carry the potentiality for bias for HCC recurrence. Interestingly, the studies conducted in USA demonstrated higher risk for HCC recurrence than Asian and European studies $(43.3 \%$ vs $28.9 \%$ vs $22.1 \%)^{36}$

\section{Patterns Of HCC Recurrence}

Studies Showed Aggressive Patterns For HCC Recurrence Reig et al observed an increased rate of $\mathrm{HCC}$ recurrence in cases that received DAAs therapy, with an aggressive course of HCC recurrence in tumor staging and future treatment options. ${ }^{4}$ Also, Renzulli et al noticed faster rate of development of HCC after DAA therapy with an aggressive course of micro vascular invasion. ${ }^{37}$ Median period, between the end of antiviral treatment and reappearance of HCC, was 82 days (ranging from $0-318$ ). ${ }^{37}$ On the similar line, Faillaci et al reported that DAAs are associated with increased aggressiveness and growth of tumor recurrence. ${ }^{38}$ An Egyptian study by El Kassas et al noticed that six of the recurring cases $(30 \%)$ that received DAAs, had more than 3 
hepatic focal lesions compared to two cases (18\%) from the non-DAA group, but these data were not statistically significant. $^{5}$

Studies Showed No Difference In HCC Recurrence Pattern According to most of the analyzed studies in a meta-analysis, Saraiya et al deduced that HCC recurrence after DAAs was not aggressive. ${ }^{36}$ In line with the previous study, Adhoute et al reported no difference in recurrence rate of HCC among two groups: group 1 with 22 patients who received DAAs following $\mathrm{HCC}$ intervention and group 2 with 49 patients who did not receive DAAs (41\% to $35 \%$ ). Also, no difference in pattern of recurrence of HCC was observed in both the groups. ${ }^{39}$ Similarly, Minami et al confirmed that increased risk of HCC recurrence and aggressiveness was not found in their study. ${ }^{40}$ According to Ikeda et al, aggressive recurrence was associated more in patients with multiple recurrences of $\mathrm{HCC}^{28}$

\section{Risk Factors For Recurrence Of HCC Post DAAs}

One of the main aims of the studies addressing the issue of $\mathrm{HCC}$ recurrence was to find out predictors of such unpleasant event (Table 1). According to various studies, different factors may be associated with HCC recurrence. Conti et al reported that increasing stiffness of the liver tissue $(>21.5 \mathrm{kPa})$ and younger age are associated significantly with recurrence of HCC after DAAs. ${ }^{22}$ Other factors like AFP, BMI, diabetes, DAA regimen and treatment modality for previous $\mathrm{HCC}$, did not affect the recurrence rate of HCC. ${ }^{22}$

According to Petta et al, AFP, bilirubin, and serum creatinine may be predictors for tumor recurrence. ${ }^{41}$ Also, Cabibbo et al showed that early recurrence rate after treatment with DAAs was $32.7 \%$ in patients with tumor size $>2.5 \mathrm{~cm}$, and $6.3 \%$ in patients with tumor size $<2.5 \mathrm{~cm} .{ }^{30}$ However, Adhoute et al did not agree with the previous studies, reporting that there were no significant risk factors that could affect the recurrence rate. ${ }^{39}$ Interestingly, El Kassas et al reported that DAAs therapy was an important risk factor for the recurrence of $\mathrm{HCC}$ along with Child-Pugh score and presence of gastroesophageal varices. ${ }^{5}$ Also, Ogawa et al demonstrated that baseline level of AFP, treatment modality, and increased number of $\mathrm{HCC}$ nodules were associated with increased $\mathrm{HCC}$ recurrence rate, while levels of ALT, AFP at the end of treatment, diabetes, and HCV genotype did not affect the recurrence rate. ${ }^{31}$ Additionally, Mashiba et al deduced that AST, PT, SVR, Fib-4, and AFP level at the end of treatment, intervention type in HCC management, and the number of focal lesions were associated with early $\mathrm{HCC}$ recurrence. ${ }^{33} \mathrm{WFA}+-\mathrm{M} 2 \mathrm{BP}$, “a biomarker for liver diseases which has a prognostic role to predict the recurrence of HCC when pre-treatment serum level $>3$ "42 was found to be significantly related to HCC recurrence. ${ }^{27}$ However, Yasui et al found that WFA+-M2BP can only predict occurrence of HCC, and AFP level > 6ng was the only predictive factor for $\mathrm{HCC}$ recurrence. ${ }^{43}$

We tried to define and tabulate the predictors and risk factors for $\mathrm{HCC}$ recurrence across the published reports (Table 1). However, a score for decision-making based on these predictors, require raw data from these studies which is perhaps difficult and beyond the scope of this review.

\section{Mechanisms And Explanations Of HCC Recurrence After DAAs}

Different hypotheses and factors have been introduced to establish the mechanism of recurrence of HCC following DAAs therapy, as it is unlikely that DAAs have a direct effect on tumor cell growth due to the decrease of malignancy incidence in HIV patients who are receiving protease inhibitors. ${ }^{3}$

Immunological factors: DAAs therapy causes dramatic decrease in the load of $\mathrm{HCV}$, with consequent dysregulation in the surveillance of immune system. ${ }^{4,44}$ Most of the studies suggest that this may be due to the difference between DAAs and IFN as the latter exhibits immune mediated antitumoral effect in eradication of $\mathrm{HCV}^{45}$ Further, IFNs exhibit an inhibitory effect on tumor angiogenesis and immune cells' activity through intrinsic effects, potentiating antimalignancy immunity. ${ }^{45}$ IFNs are actively introduced in treatment of multiple malignancies like solid and hematological tumors, even though their exact mechanism of action is not clear. ${ }^{45}$

While IFN takes a longer time to suppress viral load, DAAs can eradicate $\mathrm{HCV}$ in the initial few days of treatment which affects anti-tumoral immune control allowing tumor cells to re-emerge. ${ }^{44}$ Decreasing natural killer cells' cytotoxic activity in hepatic tissue may have a role in faster HCC development. ${ }^{44,46}$

Debes et al studied certain immune mediators present in high levels in serum before starting DAA therapy in the cases that developed HCC. ${ }^{47}$ The serum level of immune mediators was measured in 13 cases who developed HCC (three of them were recurrences) following DAA treatment. $^{47}$ They found that TNF- $\alpha$ level decreased in 
Table I Summary Of The Predictors Of HCC Recurrence Post DAAs

\begin{tabular}{|c|c|c|c|c|c|c|c|}
\hline Study & Journal/Year & Country & $\begin{array}{l}\text { Study } \\
\text { Design }\end{array}$ & $\begin{array}{l}\text { HCC } \\
\text { Recurrence } \\
\text { Rate (\%) }\end{array}$ & $\begin{array}{l}\text { Predictors Of HCC } \\
\text { Recurrence }\end{array}$ & $\begin{array}{l}\text { Median } \\
\text { Follow } \\
\text { Up } \\
\text { (Months) }\end{array}$ & $\begin{array}{l}\text { Sample } \\
\text { Size }\end{array}$ \\
\hline Conti et $\mathrm{al}^{22}$ & J Hepatol, 2016 & Italy & Retrospective & 28.8 & Age, liver stiffness & 12 & 59 \\
\hline Petta et $\mathrm{al}^{4 \mathrm{I}}$ & $\begin{array}{l}\text { Alimentary Pharmacology } \\
\text { and Therapeutics, } 2017\end{array}$ & Italy & Retrospective & 27.6 & $\begin{array}{l}\text { Baseline AFP, bilirubin and } \\
\text { creatinine levels }\end{array}$ & 18 & 58 \\
\hline Cabibbo et al ${ }^{30}$ & $\begin{array}{l}\text { Alimentary Pharmacology } \\
\text { and Therapeutics, } 2017\end{array}$ & Italy & Prospective & 21 & $\begin{array}{l}\text { Previous recurrence, } \\
\text { tumor size }\end{array}$ & 9 & 143 \\
\hline Nagata et $\mathrm{al}^{27}$ & J Hepatol, 2017 & Japan & Retrospective & 29 & SVR, AFP, WFA-M2BP & 27.6 & 83 \\
\hline El Kassas et $\mathrm{al}^{5}$ & $\begin{array}{l}\text { Journal of Viral Hepatitis, } \\
2018\end{array}$ & Egypt & Prospective & 37.7 & $\begin{array}{l}\text { Exposure to DAAs, Child-Pugh } \\
\text { score and presence of gastro } \\
\text { esophageal varices }\end{array}$ & 16 & 53 \\
\hline Ogawa et $\mathrm{al}^{31}$ & $\begin{array}{l}\text { Alimentary Pharmacology } \\
\text { and Therapeutics, } 2018\end{array}$ & Japan & Prospective & 16.5 & $\begin{array}{l}\text { Baseline level of AFP, treatment } \\
\text { modality and increased number of } \\
\text { HCC nodules }\end{array}$ & 17 & 152 \\
\hline Mashiba et al ${ }^{33}$ & PLOS ONE, 2018 & Japan & Retrospective & N/A & $\begin{array}{l}\text { Albumin, PT, AST, AFP at end of } \\
\text { antiviral treatment, duration from } \\
\text { last HCC intervention to starting } \\
\text { antiviral therapy, number of past } \\
\text { treatments for HCC, clinical HCC } \\
\text { staging, and status of SVR }\end{array}$ & 24 & 368 \\
\hline Yasui et al ${ }^{43}$ & Hepatology Research, 2018 & Japan & Retrospective & 44 & AFP level & N/A & 49 \\
\hline
\end{tabular}

Abbreviations: DAA, direct-acting antiviral agents; AFP, alpha-fetoprotein; HCC, hepatocellular carcinoma; SVR, sustained virological response; NA, not available.

the $4^{\text {th }}$ week following DAA therapy in the cases who did not develop HCC after DAA therapy in the follow-up period, while it was constant in the cases who developed HCC. So, higher levels of TNF- $\alpha$ could have a role in $\mathrm{HCC}$ occurrence and recurrence following DAA therapy. ${ }^{47}$

Genetic factors: other factors like vascular endothelial growth factor (VEGF) which induce angiogenesis, probably have a significant role in HCC development following DAA therapy. ${ }^{48}$ Also, patients with new or recurrent HCC may have higher levels of angiopoietin-2 in contrast to non-HCC cases, as shown by Faillaci et al who studied RNA from liver tissue in cases who received DAA therapy. They also analyzed the role of VEGF as an inducer in cases with splanchnic collaterals and severe liver fibrosis. These patients have highly activated neoangiogenesis pathways in hepatic tissues as confirmed by high level of angiopoietin-2. ${ }^{48}$

Reduced levels of microRNA 122 (miR-122) in serum was observed in HCV patients who received DAAs therapy following SVR. ${ }^{49}$ MiR-122 is known to be one of the most available miRNAs in hepatic tissues and has an inhibitory effect on the development of HCC. ${ }^{50}$ So, decreased level of miR-122 in patients receiving DAAs may lead to higher risk of $\mathrm{HCC}$ recurrence. ${ }^{51}$

Epidemiological factors: new DAA therapy gives us a chance to treat the more advanced cirrhosis population in comparison to the era of IFN with higher cure rates in cirrhotic patients. ${ }^{52,53}$ Therefore, HCC incidence may be markedly higher in the era of DAA therapy. ${ }^{35}$ It is known that $\mathrm{HCC}$ development may increase in patients with a higher degree of liver fibrosis and with the increasing age of the patients. ${ }^{54}$ So, there is a higher risk of HCC development in cases with liver cirrhosis receiving DAAs therapy and this has led to 3-folds higher HCC incidence in certain meta-analyses. ${ }^{35}$ Furthermore, incidence of HCC in cirrhotic cases will also depend on multiple factors, including detection mechanisms, time of follow-up, extent of follow-up period, and schedule of screening. ${ }^{55,56}$ Cases of HCC, which were missed before starting DAA therapy, may be considered as de novo after treatment, if follow-up timing for detection of HCC started at the end of DAA therapy and also if the duration is short in the new era of 
DAAs, in comparison to the longer duration in the era of IFN. ${ }^{35}$ Moreover, an existing cohort effect in the previous studies may be present, which could be the cause of the higher initial HCC incidence that occurs because of the higher HCC risk profile at baseline in cirrhotic cases receiving DAA therapy, in addition to the undiagnosed pretreatment $\mathrm{HCC}$ cases that will be detected at early post treatment period. ${ }^{57}$

Also, there is a bias in defining the risk of $\mathrm{HCC}$ recurrence in cases receiving curative $\mathrm{HCC}$ management. Noted areas of bias were timing, follow-up schedule, and definition criteria of HCC curative populations. ${ }^{35}$ This becomes more complex with difficulty in distinguishing between recurrent $\mathrm{HCC}$ cases and de novo ones. ${ }^{58}$ Also, there was significant variation in the time period of followup in relation to HCC diagnosis and starting DAAs therapy. Lower recurrence rate of HCC incidence related to longer follow-up time, was close to that noticed in evaluation of occurrence of HCC; which strongly suggests the definition of cohort effect in patients with high risk of HCC recurrence. ${ }^{35}$

A more potent cohort effect is noticed in $\mathrm{HCV}$ cure field, which is explained by improving liver status in cirrhotic cases with decreased individual risk, especially noted with occurrence of HCC. ${ }^{59}$ Pretreatment of cirrhotic cases treated with DAAs still carry residual risk of HCC and this necessitates continued screening of $\mathrm{HCC}$ in all patients until the target subgroups show negligible risk. ${ }^{35}$

However, these studies need to be confirmed and further studies are required to know and identify the exact mechanisms leading to liver carcinogenesis after DAAs therapy.

\section{Limitations Of The Published Studies}

A lot of the studies which discussed the risk of HCC recurrence were retrospective single armed studies, with wide heterogenicity regarding HCC treatment and followup period. Also, HCC recurrence can be affected by the type of HCC intervention "curative or palliative", and hence can vary due to the short period of follow-up as in recent studies. Therefore, we need large, multicenter prospective studies with control groups and longer periods of follow-up to identify the exact risk of HCC following DAAs treatment and to know the benefit of these DAAs in preventing the development and recurrence of HCC.

\section{Conclusion}

All the available studies done so far are conflicting about whether DAAs can predispose to HCC or not. Therefore, a definitive conclusion cannot be reached. Henceforth, we need to fully understand new DAAs and assess the risk-benefit ratio of using them in cases with HCC history. We can, however, conclude that we should wait for at least $6-12$ months before starting DAAs post HCC treatment.

\section{Disclosure}

The authors report no conflicts of interest in this work.

\section{References}

1. Holah NS, El-Azab DS, Aiad HA, Sweed DM. Hepatocellular carcinoma in Egypt: epidemiological and histopathological properties. Menoufia Med J. 2015;28:718-724.

2. Tanimoto $\mathrm{Y}$, Tashiro $\mathrm{H}$, Aikata $\mathrm{H}$, et al. Impact of pegylated interferon therapy on outcomes of patients with hepatitis $\mathrm{C}$ virusrelated hepatocellular carcinoma after curative hepatic resection. Ann Surg Oncol. 2012;19(2):418-425. doi:10.1245/s10434-0111866-1

3. Reig M, Mariño Z, Perelló C, et al. High Rate of early tumor recurrence in patients with $\mathrm{HCV}$-related HCC undergoing interferon-free therapy. $J$ Hepatol. 2016;65(4):719-726. doi:10.1016/j.jhep.2016.04.008

4. Reig M, Mariño Z, Perelló C, et al. Tumor recurrence after interferon-free treatment for hepatitis $\mathrm{C}$ in patients with previously treated hepatocellular carcinoma discloses a more aggressive pattern and faster tumor growth. J Hepatol. 2017;66:S20. doi:10.1016/S01688278(17)30302-1

5. El Kassas M, Funk AL, Salaheldin M, et al. Increased recurrence rates of hepatocellular carcinoma after DAA therapy in a hepatitis C-infected egyptian cohort: a comparative analysis. J Viral Hepat. 2018;25(6):623-630. doi:10.1111/jvh.2018.25.issue-6

6. Cheng Z, Yang P, Qu S, et al. Risk factors and management for early and late intrahepatic recurrence of solitary hepatocellular carcinoma after curative resection. Hpb J. 2015;17:422-427. doi:10.1111/hpb.12367

7. Arnaoutakis D, Mavros M, Shen F, et al. Recurrence patterns and prognostic factors in patients with hepatocellular carcinoma in noncirrhotic liver: a multi-institutional analysis. Ann Surg Oncol. 2014;21(1):147-154. doi:10.1245/s10434-013-3211-3

8. Imai Y, Tamura S, Tanaka H, et al. Reduced risk of hepatocellular carcinoma after interferon therapy in aged patients with chronic hepatitis $\mathrm{C}$ is limited to sustained virological responders. $J$ Viral Hepat. 2010;17(3):185-191. doi:10.1111/jvh.2010.17.issue-3

9. $\mathrm{Xu} \mathrm{C}$, Luo $\mathrm{L}$, Yu Y, et al. Screening therapeutic targets of ribavirin in hepatocellular carcinoma. Oncol Lett. 2018;15(6):9625-9632. doi: 10.3892/ol.2018.8552

10. Hiroaki H, Nouso K, Kobayashi Y, et al. Effect of pegylated interferon therapy on intrahepatic recurrence after curative treatment of hepatitis $\mathrm{C}$ virus-related hepatocellular carcinoma. Int J Clin Oncol. 2011;16 (3):210-220.

11. Shuhei N, Akihiro T, Shoji K.Effect of long-term postoperative interferon therapy on intrahepatic recurrence and survival rate after resection of hepatitis $\mathrm{C}$ virus-related hepatocellular carcinoma. Intervirology. 2005;48(1):71-75. doi:10.1159/000082098

12. Miyake Y, Takaki A, Iwasaki Y, Yamamoto K. Meta-analysis: interferon-alpha prevents the recurrence after Curative treatment of hepatitis C virus-related hepatocellular carcinoma. J Viral Hepat. 2010;17 (4):287-292. doi:10.1111/jvh.2010.17.issue-4 
13. Yoshida H, Shiratori Y, Moriyama M, et al. Interferon therapy reduces the risk for hepatocellular carcinoma: national surveillance program of cirrhotic and non-cirrhotic patients with chronic hepatitis C in Japan. Ann Intern Med. 1999;131:174-181. doi:10.7326/0003-4819-131-3199908030-00003

14. Takeaki S, Mitsuda A, Koda M, et al. Interferon alpha inhibits intrahepatic recurrence in hepatocellular carcinoma with chronic hepatitis C: a pilot study. Hepatol Res. 2001;20(3):301-311. doi:10.1016/S1386-6346 (00)00148-0

15. Naoya K, Ogasawara S, Chiba T, et al. Sustained virologic response achieved after curative treatment of hepatitis $\mathrm{C}$ virus-related hepatocellular carcinoma as an independent prognostic factor. $J$ Gastroenterol Hepatol. 2015;30(7):1197-1204. doi:10.1111/jgh.12925

16. Cheol JS, Aikata H, Katamura Y, et al. Effects of a 24-week course of interferon- $\alpha$ therapy after curative treatment of hepatitis C virus-associated hepatocellular carcinoma. World J Gastroenterol. 2013;13 (40):5343-5350.

17. El-Serag HB, Kanwal F, Richardson P, Kramer J. Risk of hepatocellular carcinoma after sustained virologic response in veterans with HCVinfection. Hepatology. 2016;64:130-137. doi:10.1002/hep.28535

18. Kensaku S, Kayashima H, Iguchi T, et al. Characterization of hepatocellular carcinoma developed after achieving sustained virological response to interferon therapy for hepatitis C. J Surg Oncol. 2009;99 (1):32-37. doi:10.1002/jso.21176

19. Elbaz T, El-Kassas M, Esmat G. New era for management of chronic hepatitis $\mathrm{C}$ virus using direct antiviral agents: a review. $J$ Adv Res. 2015;6(3):301-310. doi:10.1016/j.jare.2014.11.004

20. El Kassas M, Elbaz T, Hafez E, Esmat G. Safety of direct antiviral agents in the management of hepatitis C. Expert Opin Drug Saf. 2016;15(12):1643-1652. doi:10.1080/14740338.2017.1240781

21. Reig M, Boix L, Mariño Z, Torres F, Forns X, Bruix J. Liver cancer emergence associated with antiviral treatment: an immune surveillance failure? Semin Liver Dis. 2017;37:109-118. doi:10.1055/s0037-1601349

22. Conti F, Federica B, Alessandra S, et al. Early occurrence and recurrence of hepatocellular carcinoma in HCV-related cirrhosis treated with direct-acting antivirals. J Hepatol. 2016;65(4):727-733. doi:10.1016/j.jhep.2016.06.015

23. Kolly P, Waidmann O, Vermehren J, et al. Hepatocellular carcinoma recurrence after direct antiviral agent treatment: a European multicentre study. J Hepatol. 2017;67:876-878. doi:10.1016/j.jhep.2017.07.007

24. Yang JD, Aqel BA, Pungpapong S, Gores GJ, Roberts LR, Leise MD. Direct acting antiviral therapy and tumor recurrence after liver transplantation for hepatitis C-associated hepatocellular carcinoma. $J$ Hepatol. 2016;65(4):859-860. doi:10.1016/j.jhep.2016.06.023

25. Granata R, Di Costanzo G, Sanduzzi Zamparelli M, et al. Hepatocellular carcinoma recurrence rate in $\mathrm{HCV}$ infected patients treated with direct antiviral agents. A single center experience. Digestive Liver Dis 2017;49 (1):e45-46. doi:10.1016/j.dld.2017.01.094

26. Victor V, Pradat P, Hartig-Lavie K, et al. Direct-acting antiviral therapy decreases hepatocellular carcinoma recurrence rate in cirrhotic patients with chronic hepatitis C. Liver Int. 2017;37(8):11221127. doi:10.1111/liv.13456

27. Hiroko N, Nakagawa M, Asahina Y, et al. Effect of interferon-based and -free therapy on early occurrence and recurrence of hepatocellular carcinoma in chronic hepatitis C. J Hepatol. 2017;67(5):933939. doi:10.1016/j.jhep.2017.05.028

28. Ikeda K, Yusuke K, Masahiro K, et al. Direct-acting antivirals decreased tumor recurrence after initial treatment of hepatitis $\mathrm{C}$ virus-related hepatocellular carcinoma. Dig Dis Sci. 2017;62 (10):2932-2942. doi:10.1007/s10620-017-4739-z

29. Sangiovanni A, Alimenti E, Biganzoli E. IFN-free DAA treatment of cirrhotic HCV patients with or without history of HCC: a multicenter prospective trial in Italy. Hepatology. 2017;66(Supplement 1 PG734A-735A):734A-735A.
30. Cabibbo G, Petta S, Calvaruso V, et al. Is early recurrence of hepatocellular carcinoma in HCV cirrhotic patients affected by treatment with direct-acting antivirals? A prospective multicentre study. Aliment Pharmacol Ther. 2017;46(7):688-695. doi:10.1111/apt.14256

31. Ogawa E, Furusyo N, Nomura H, et al. Short-term risk of hepatocellular carcinoma after hepatitis $\mathrm{C}$ virus eradication following directacting anti-viral treatment. Aliment Pharmacol Ther. 2018;47(1):104113. doi:10.1111/apt. 14380

32. Ogawa E, Furusyo N, Kajiwara E, et al. Efficacy of pegylated interferon alpha- $2 \mathrm{~b}$ and ribavirin treatment on the risk of hepatocellular carcinoma in patients with chronic hepatitis C: a prospective, multicenter study. $J$ Hepatol. 2013;58(3):495-501. doi:10.1016/j.jhep.2012.10.017

33. Mashiba T, Kouji J, Masayuki K, et al. Does interferon-free direct-acting antiviral therapy for hepatitis $\mathrm{C}$ after curative treatment for hepatocellular carcinoma lead to unexpected recurrences of HCC? A multicenter study by the Japanese Red Cross Hospital Liver Study Group. PLoS One. 2018;13(4):1-13. doi:10.1371/journal.pone.0194704

34. ANRS collaborative study group on hepatocellular carcinoma (ANRS CO22 HEPATHER, CO12 CirVir and CO23 CUPILT cohorts) Electronic address: stanislas.pol@aphp.fr. Lack of evidence of an effect of direct-acting antivirals on the recurrence of hepatocellular carcinoma: data from three ANRS cohorts. J Hepatol. 2016;65::734740. doi:10.1016/j.jhep.2016.05.045

35. Waziry R, Behzad H, Jason G, et al. Hepatocellular carcinoma risk following direct-acting antiviral HCV therapy : a systematic review. $J$ Hepatol. 2017;67(6):1204-1212. doi:10.1016/j.jhep.2017.07.025

36. Saraiya N, Yopp AC, Rich NE, Odewole M, Parikh ND, Singal AG. Systematic review with meta-analysis: recurrence of hepatocellular carcinoma following direct-acting antiviral therapy. Aliment Pharmacol Ther. 2018;48(2):127-137. doi:10.1111/apt.14823

37. Renzulli M, Buonfiglioli F, Conti F, et al. Imaging features of microvascular invasion in hepatocellular carcinoma developed after directacting antiviral therapy in HCV-related cirrhosis. Eur Radiol. 2018; 28:506-513. doi:10.1007/s00330-017-5033-3

38. Faillaci F, Luca M, Rosina C, et al. Liver angiopoietin-2 is a key predictor of de novo or recurrent hepatocellular cancer after hepatitis C virus direct-acting antivirals. Hepatology. 2018;68(3):1010-1024. doi:10.1002/hep. 29911

39. Adhoute X, Guillaume P, Jean Luc R, et al. Hepatocellular carcinoma recurrence in hepatitis $\mathrm{C}$ virus-related cirrhosis treated with directacting antivirals: a case-control study. Eur J Gastroenterol Hepatol. 2018;30(4):368-375. doi:10.1097/MEG.0000000000001082

40. Minami T, Ryosuke T, Ryo N, et al. The impact of direct-acting antivirals on early tumor recurrence after radiofrequency ablation in hepatitis C-related hepatocellular carcinoma. J Hepatol. 2016;65 (6):1272-1273. doi:10.1016/j.jhep.2016.07.043

41. Petta S, Cabibbo G, Barbara M, et al. Hepatocellular carcinoma recurrence in patients with curative resection or ablation: impact of $\mathrm{HCV}$ eradication does not depend on the use of interferon. Aliment Pharmacol Ther. 2017;45(1):160-168. doi:10.1111/apt.13821

42. Zhou D, Yaqin W, Wen Z, Lu C, Jiajie L. WFA(+) -M2BP: a novel biomarker with diagnostic and therapeutic implications in liver diseases. Liver Int. 2016;36:612. doi:10.1111/liv.13024

43. Yasui Y, Masayuki K, Yasuyuki K, et al. Wisteria floribunda agglutinin-positive mac-2 binding protein predicts early occurrence of hepatocellular carcinoma after sustained virologic response by direct-acting antivirals for hepatitis C virus. Hepatol Res. 2018;48 (13):1131-1139. doi:10.1111/hepr.13233

44. Kanda T, Matsuoka S, Moriyama M. Early occurrence and recurrence of hepatocellular carcinoma in hepatitis C virus-infected patients after sustained virological response. Hepatol Int. 2018;12:90-93. doi:10.1007/s12072-018-9862-1

45. Pasquali S, Mocellin S. The anticancer face of interferon alpha (IFNalpha): from biology to clinical results, with a focus on melanoma. Curr Med Chem. 2010;17:3327-3336. doi:10.2174/092986710793176393 
46. Chu PS, Nakamoto N, Taniki N, et al. On-treatment decrease of NKG2D correlates to early emergence of clinically evident hepatocellular carcinoma after interferon-free therapy for chronic hepatitis C. PLoS One. 2017;12:e0179096. doi:10.1371/journal.pone.0179096

47. Debes JD, van Tilborg M, Groothuismink ZMA, et al. Levels of cytokines in serum associate with development of hepatocellular carcinoma in patients with $\mathrm{HCV}$ infection treated with direct-acting antivirals. Gastroenterology. 2018;154:515-517.e. doi:10.1053/j.gastro.2017.10.035

48. Villani R, Facciorusso A, Bellanti F, et al. DAAs rapidly reduce inflammation but increase serum VEGF level: a rationale for tumor risk during anti-HCV treatment. PLoS One. 2016;11:e0167934. doi:10.1371/journal.pone.0167934

49. Waring JF, Dumas EO, Abel S, et al. Serum miR-122 may serve as a biomarker for response to direct acting antivirals: effect of paritaprevir/R with dasabuvir or ombitasvir on miR-122 in HCV-infected subjects. J Viral Hepat. 2016;23:96-104. doi:10.1111/jvh.12470

50. Nakao K, Miyaaki H, Ichikawa T. Antitumor function of microRNA122 against hepatocellular carcinoma. J Gastroenterol. 2014;49:589593. doi:10.1007/s00535-014-0932-4

51. Liu $\mathrm{CH}$, Kao JH. Hepatocellular carcinoma recurrence after interferon-free direct acting antiviral treatment for chronic hepatitis $\mathrm{C}$ virus infection: fact or fiction? Transl Cancer Res. 2016;5:S192195. doi:10.21037/tcr.2016.07.32

52. Poordad F, Hezode C, Trinh R, et al. ABT-450/r ombitasvir and dasabuvir with ribavirin for hepatitis C with cirrhosis. $N$ Engl J Med. 2014;370:1973-1982. doi:10.1056/NEJMoa1402869
53. Poordad F, Schiff ER, Vierling JM, et al. Daclatasvir with sofosbuvir and ribavirin for hepatitis $\mathrm{C}$ virus infection with advanced cirrhosis or post liver transplantation recurrence. Hepatology. 2016;63:14931505. doi:10.1002/hep.v63.5

54. Waziry R, Grebely J, Amin J, et al. Trends in hepatocellular carcinoma among people with $\mathrm{HBV}$ or $\mathrm{HCV}$ notification in Australia (2000-2014). J Hepatol. 2016;65:1086-1093. doi:10.1016/j.jhep. 2016.08.010

55. Mancebo A, González-Diéguez ML, Navascués CA, et al. Adherence to a semiannual surveillance program for hepatocellular carcinoma in patients with liver cirrhosis. J Clin Gastroenterol. 2017;51:557-563. doi:10.1097/MCG.0000000000000734

56. Bruix J, Sherman M. Management of hepatocellular carcinoma: an update. Hepatology. 2011;53:1020-1022. doi:10.1002/hep.24199

57. Nault J-C, Colombo M. Hepatocellular carcinoma and direct acting antiviral treatments: controversy after the revolution. J Hepatol. 2016;65:663-665. doi:10.1016/j.jhep.2016.07.004

58. Marubashi S, Sakon M. "Local recurrence" is not equal to "Local dissemination" after resection for hepatocellular carcinoma. $J$ Hepatol. 2016;65:1061. doi:10.1016/j.jhep.2016.06.030

59. Knop V, Hoppe D, Welzel T, et al. Regression of fibrosis and portal hypertension in $\mathrm{HCV}$-associated cirrhosis and sustained virologic response after interferon-free antiviral therapy. $J$ Viral Hepat. 2016;23:994-1002. doi:10.1111/jvh.12578
Journal of Hepatocellular Carcinoma

\section{Publish your work in this journal}

The Journal of Hepatocellular Carcinoma is an international, peerreviewed, open access journal that offers a platform for the dissemination and study of clinical, translational and basic research findings in this rapidly developing field. Development in areas including, but not limited to, epidemiology, vaccination, hepatitis therapy, pathology and molecular tumor classification and prognostication are all considered for publication. The manuscript management system is completely online and includes a very quick and fair peer-review system, which is all easy to use. Visit http://www.dovepress.com/ testimonials.php to read real quotes from published authors. 